\title{
AOOT 1961
}

\section{REVUE}

\section{INTERNATIONA LE}

DE LA

\section{CROIX-ROUGE}

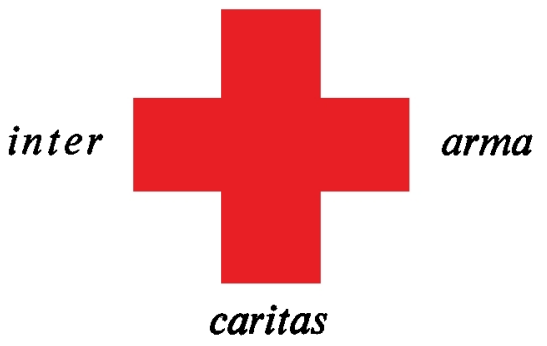

COMITE INTERNATIONAL DE LA CROIX-ROUGE GENÈVE 


\title{
COMITÉ INTERNATIONAL DE LA CROIX-ROUGE
}

\author{
à Genève
}

MM. LEOPOLD BOISSIER, docteur en droit, professeur honoraire de l'Université de Genève, ancien secrétaire général de l'Union interparlementaire, président (1946) 1

JACQUES CHENEVIERE, docteur ès lettres h.c., tice-président d'honneur (1919)

M"* LUCIE ODIER, ex-chef du Service des infirmières-visiteuses de la Section genevoise de la Croix-Rouge suisse (1030)

MM. CARL J. BURCKHARDT, docteur en philosophie, ancien ministre de Suisse en France (1933)

MARTIN BODMER, docteur en philosophie h.c., vice-prósident (1940)

ERNEST GLOOR, docteur en médecine, vice-president (1945)

PAUL RUEGGER, ancien ministre de Suisse en Italie et en Grande-Bretagne, membre de la Cour Permanente d'Arbitrage (1048), en congé

RODOLFO OLGIATI, docteur en médecine h.c., ancien directeur du Don suisse (1949)

M11 MARGUERITE VAN BERCHEM, ancien chef de service de l'Agence centrale des prisonniers de guerre (1051)

MM. FRÉDF́RIC SIORDET, avocat, conseiller du Comité international de la Croix-Rouge de 1043 à 1051 , (1051)

GUILLAUME BORDIER, ingénieur dipl. E.P.F., M.B.A. Harvard, banquier (1955)

ADOLPHF FRANCESCHETTI, docteur en médecine, professeur de clinique ophtalmologique a l'Université de Genève (1958)

HANS BACHMANN, docteur en droit, secrétaire généra! ad;oint du Comité international de la CroixRouge de 1944 a 1946 (1958)

JACQUES FREYMOND, docteur ès lettres, directeur de l'Institut universitaire de hautes études internationales, professeur à l'Université de Genève (1959)

DIETRICH SCHINDLER, docteur en droit (1901)

SAMUEL GONARD, colonel commandant de corps, ancien professeur à l'Ecole polytechnique fédérale (1961)

HANS MEULI, docteur en médecine, colonel brigadier, ancien chef du Service de santé de l'armée suisse (1931)

\section{Divection:}

MM. ROGER GALLOPIN, docteur en droit, directeur exécutif

JEAN S. PICTET, docteur en droit, directeur des Affaires générales

EDOUARD de BONDELI, sous-directeur, Services financiers et administratifs

CLAUDE PILLOUD, sous-directeur, Service juridique

1 Les années indiquées dans les parenthèses désignent les dates de nomination des membres du Comité ioternational. 


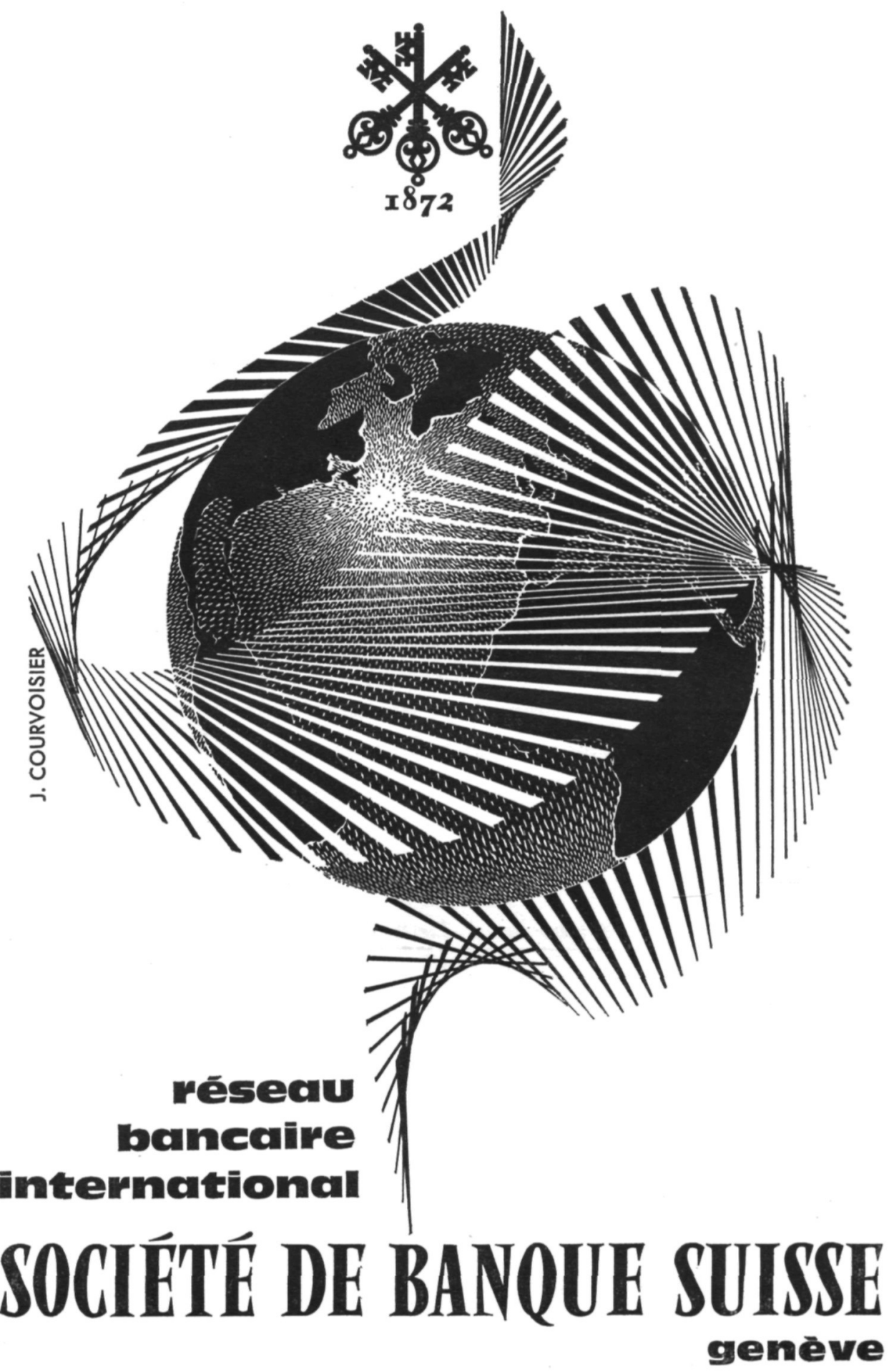




\section{SOCIETE FIDUCIAIRE ROMANDE OFOR S.A.}

9, rue d'Italie, Genève - Tél. 243293

$\downarrow$ Expertises, revisions et organisations comptables

— Domiciliation et administration de sociétés

४ Assainissements et liquidations

$\downarrow$ Interventions et conseils en matière fiscale

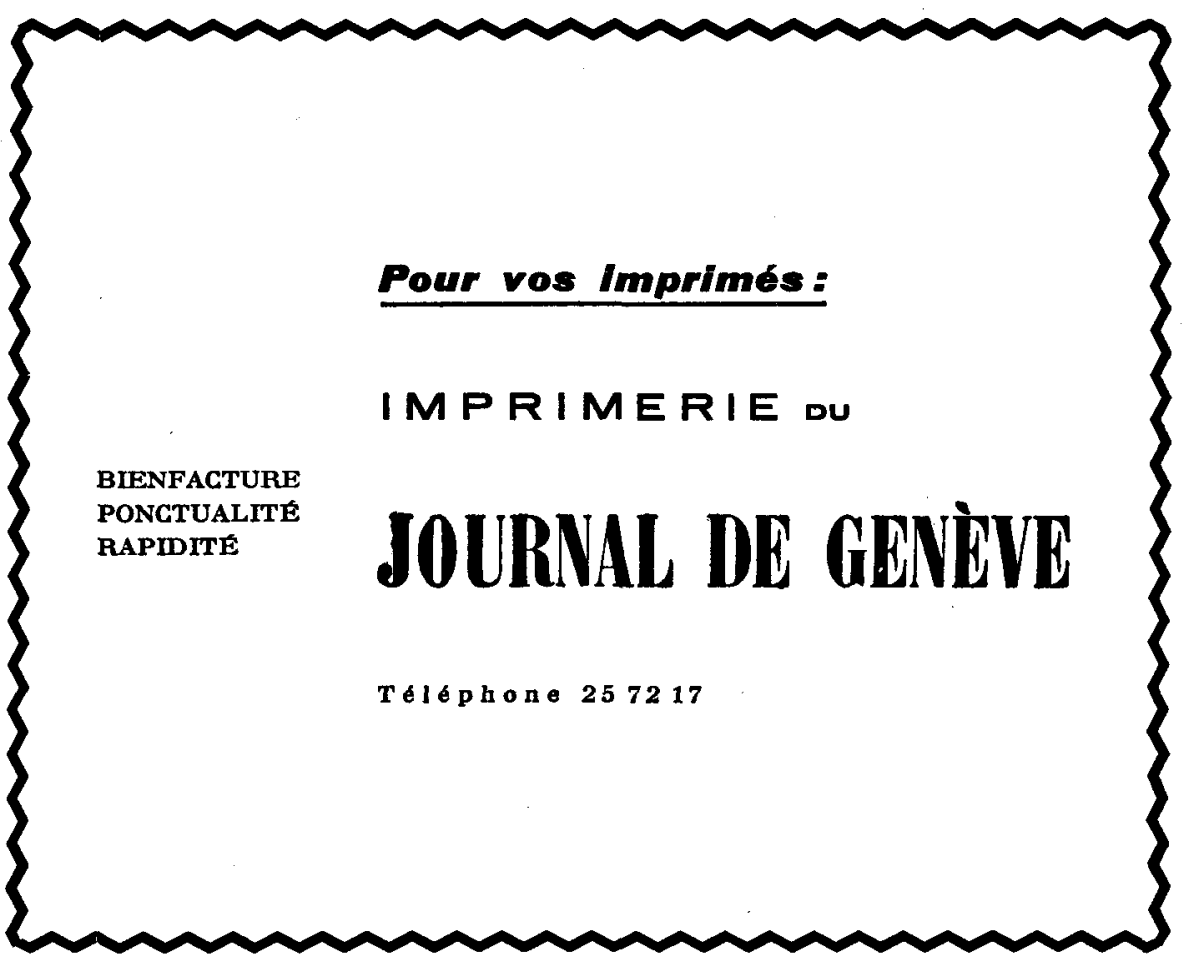




\section{REVUE INTERNATIONALE DE LA CROIX-ROUGE}

QUARANTE-TROISIEMME ANNEE - NO 512

AOOT 1961

\section{SOMMAIRE}

René Dovaz: La radiodiffusion au service du Comité international de la Croix-Rouge . . . . . . . . . . . . . . . . . . . . . 365

Drs Boško Jakovljević et Jovica Patrnogič: Nécessité urgente de l'application des règles du droit humanitaire dans les conflits armés dits internes.

\section{COMITE INTERNATIONAL DE LA CROIX-ROUGE}

Nouvelles d'ici et de partout . . . . . . . . . . . . . 386

Le Comité international et les événements de Bizerte . . . . . 399

\section{NOUVELLES DE SOCIÉTÉS NATIONALES}

Ethiopie . . . . . . . . . . . . . . 401

Portugal ................... . 403

U.R.S.S. . . . . . . . . . . . . . . 406

\section{CHRONIQUE}

L'action de la Ligue des Sociétés de la Croix-Rouge en faveur des paralysés marocains . . . . . . . . . . . . . . . 408

\section{A TRAVERS LES REVUES}




\title{
EDITION ANGLAISE DE LA REVUE
}

Une édition en langue anglaise paraît chaque mois sous le titre:

\section{International Review of the Red Cross}

Elle est en principe identique à l'édition française, et peut être obtenue aux mêmes conditions.

\section{SUPPLEMENTS DE LA REVUE}

\author{
EN LANGUE ESPAGNOLE
}

Informaciones del mundo entero. - El Comité internacional y los acontecimientos de Bizerta.

EN LANGUE ALLEMANDE

Nachrichten aus Nah und Fern. - Das Internationale Komitee und die Ereignisse in Biserta.

LA

REVUE INTERNATIONALE DE LA CROIX-ROUGE est publiée chaque mois par le Comité international de la Croix-Rouge

7, avenue de la Paix, Genève (Suisse) - Compte de chèques postaux I, 1767 Abonnement un an: Fr. 20,-: le numéro Fr. 2,-

Pour les pays suivants: Algérie, Allemagne, Autriche, Belgique, Danemark, Finlande, France, Italie, Luxembourg, Maroc, Norvège, Pays-Bas, Portugal, Sarre, Suède, Tunisie, Vatican, on peut s'abonner auprès des bureaux de poste (fr. s. 20,50)

Rédaction : JEAN-G. LOSSIER 